\title{
An Integral Approach for Understanding the Bioactive Small Molecules in Cardiovascular Diseases
}

\author{
Yifan $\mathrm{Chen}^{1}$ and jun $\mathrm{pu}^{1}$ \\ ${ }^{1}$ Shanghai Jiao Tong University School of Medicine Affiliated Renji Hospital
}

May 4, 2020

\begin{abstract}
Cardiovascular disease (CVD) remains a leading cause of mortality worldwide. In recent years, bioactive small molecules (BSMs) have been demonstrated to play crucial roles in regulating different pathological process of cardiovascular diseases, which provides potential clinical applications. Here, we summarized five main chrematistics of bioactive small molecules in the field of cardiovascular to depict a whole regulation network of these small molecules and discuss the potential clinical therapies of bioactive small molecules including drug development and biomarkers in cardiovascular diseases.
\end{abstract}

\section{Introduction}

Despite advances in clinical intervention and drug treatment, cardiovascular disease (CVD) remains a leading cause of mortality worldwide except Africa [1]. According to the Global Burden of Disease Study 2016, the CVD resulted in 17.6 million deaths in 2016, which increased by $14.5 \%$ between 2006 and 2016 [2]. As the prevalence of chronic diseases increases steeply with age, it is crunch time for us to understand the biology of CVD and make effort to find new strategies for clinical intervention.

However, the pathological process of cardiovascular disease is very complicated, including the control of oxidative stress, inflammation, angiogenesis, cell proliferation, atherosclerosis, tissue repairing and cardiac electrophysiology. In addition to the differential expression of genes, the regulation network of these pathological changes also involves the multiple molecules such as miRNA, lncRNA, and exosomes. Different molecules form a thorough interaction network to jointly regulate the development of CVD.

Importantly, mounting evidence suggested significant roles of bioactive small molecules (BSMs) in cardiovascular system. BSMs, which include gaseous signal molecules, small molecules proteins, active peptides, active amino acids and derivatives, amines, lipids, metal ions, etc., play pivotal roles as signaling messengers maintaining cardiovascular homeostasis and in the pathogenesis of many cardiovascular disorders $[3,4]$. Therefore, elucidating the dynamic changes and mutual regulatory networks of BSMs and studying their physiological functions of the heart will help reveal important molecular events and explore potential drug targets in the development of cardiovascular disease. It is no exaggeration to say that BSMs provide new insights for the prevention and treatment of cardiovascular diseases.

This review summarizes five main features of BSMs in cardiovascular research after a short introduction about the current understanding of BSMs and then analyses current advances and challenges of BSMs in drug discovery and other application in cardiovascular diseases.

\section{Bioactive small molecules in cardiovascular diseases}

As we all know, there are universal laws of genetics in nature. The classic genetics paradigm of DNA-RNAprotein has led the research of biomedicine. At present, with the development of high-throughput technology, this idea has evolved into the research of genomics, transcriptomics, and proteomics, and important progress 
has been made in this regard. However, the progress of these three types of omics is still focused on the changes inside the cell. In fact, external cell-cell interactions and communication networks play a crucial role in the course of most heart diseases. For example, heart failure is the ultimate development of all heart diseases [5]. Heart function has evolved from decompensated changes to decompensated changes, and the heart structure has progressed from hypertrophic heart disease to dilated heart disease. This pathological myocardial remodeling involves not only myocardial cells themselves, but also various cells such as immune cells, myocardial fibroblasts, and vascular endothelial cells [6]. The expression levels of a variety of different endogenous BSMs have been shown to change significantly in tissue damage and play an important regulatory role in the microenvironment interaction network through neuro-endocrine-immune regulation [7].

At present, the definition of BSMs is not very clear. Generally, BSMs are defined as organic matter with a molecular weight below pharmacology, and can regulate a variety of biological processes [8]. Traditionally, these active small molecules were divided into two categories, endogenous and exogenous. Endogenous BSMs include metabolic small molecules derived from lipids, proteins, nucleic acids, and sugars. They can be produced by the human body to maintain and regulate the internal homeostasis. Exogenous BSMs refer to some natural organic small molecules, which cannot be produced by the body itself, but can be extracted from nature and can regulate the pathophysiology of the body, such as cycloastragenol. As a natural product from the herbAstragalus mongholicus Bunge (Huangqi), it served as a cardiac protecting factor via inhibiting inflammation and cardiomyocyte apoptosis in acute myocardial infarction rats [9]. Meanwhile, there is still a controversy whether the organic synthetic small molecules belong to BSMs. In this review, we still classify these artificial small molecules as BSMs, because they can also regulate in physiological procession and even some would be measured in the human body afterwards.

Another concern is that there is no uniform standard for the classification of BSMs because of the various types and diverse biofunctions. Most researchers tend to classify by chemical structure (Table 1 ), which is widely used in the field of drug design and development [10]. Another classification strategy is to cluster small molecules based on the biological processes involved, for example, to cluster small molecules based on different biological processes involved in inflammatory response, oxidative stress, ion channels, cell metabolism, and angiogenesis. From this, it will be useful to draw the instructive interaction network atlas among different BSMs with the help of function enrichment and cluster analysis in the future.

\section{Five main characteristics of BSMs regulating cardiovascular diseases}

The difficulty in classification comes from our inadequate research on the characteristics of BSMs. In fact, although the specific definition of BSMs remains uncertain, there are many important commonalities between different BSMs. Figure 1 summarizes the five major characteristics of BSMs in cardiovascular disease research, including low molecular weight and simple structure, wide distribution, diverse biological effects, rapid synthesis and metabolism, and low immunogenicity. These five characteristics provide a solid foundation for understanding the biology of BSMs to participate in and maintain homeostasis. In the following part, we will discuss how these characteristics of BSMs affect the occurrence and development of cardiovascular disease.

\subsection{Low molecular weight, simple structure and low immunogenicity of BSMs}

The significance of studying BSMs is that they are good potential drugs. As we all know, most of the drugs currently used in clinical practice are small-molecule drugs, such as antibiotics [11] and small-molecule kinase inhibitors targeted cancer [12]. In pharmacology, small-molecule drugs have very different characteristics from large-molecule drugs (Table 2 ). Low molecular weight, simple structure and low immunogenicity are the most obvious differences between these two types of drugs. Low molecular weight and simple structure are the prerequisites for drug screening and design. Achievement of recommended levels of low-density lipoprotein cholesterol (LDL-C) is an effective strategy to reduce the lethal risk of atherosclerotic cardiovascular disease (ASCVD) [13]. Although statins are the recommended first-line medication, a substantial number of highrisk patients are intolerance to statins or can't achieve the recommended level of LDL-C despite taking the maximum-tolerated dose of statins. It's urgent to develop next generation lipid-lowering drugs. And small- 
molecule drugs have attracted much attention because of their simple structure to be easy screened and designed. Ezetimibe and bempedoic acid (ETC-1002), which has just been approved by the FDA, are both oral small-molecule drugs to lower LDL-C effectively and stably. As a small molecule, Ezetimibe targets the Niemann-PickC1-like protein 1 receptor in the brush border of the small intestine to interfere exogenous cholesterol absorption resulting lower LDL-C levels in patients [14]. While, bempedoic acid is a new small molecule agent targeting ATP Citrate Lyase to reduce LDL-C levels and it was shown to inhibit LDL-C synthesis in animal experiments [15]. Recently new evidence has shown that the addition of bempedoic acid can reduce LDL-C level significantly among patients receiving maximally tolerated statins over 12 weeks in the phase III clinical trials [16] and more trials on its safety and efficacy are still ongoing [17].

In addition, small-molecule drugs are often simply synthesized and promoted through organic chemistry so that the cost is relatively low. Proprotein convertase subtilisin / kexin type 9 (PCSK9) is a new-reported protease which can bind low-density lipoprotein receptors (LDLRs) and consequently cause them degradation to regulate lipoprotein metabolism. According to this novel target, two biomacromolecules, alirocumab and evolocumab, were approved in 2015. Although these two monoclonal antibodies have proven to be effective [18], biomacromolecules are complicated to prepare and difficult to preserve, which lead to high cost and low long-term compliance.

At the same time, the disadvantage of the high immunogenicity carried by these antibody-based therapies cannot be ignored [19]. This kind of immunogenicity means more neoantigen presentation and even excessive autoimmune responses, which represents as the side effects like allergies and chronic inflammation. Therefore, small molecule inhibitors targeting PCSK9 are being actively developed as alternative currently. Inclisiran, a new small molecule siRNA drug, is considered a potential drug candidate and its phase III clinical trial ORION-1 shows that taking inclisiran can continuously reduce LDL-C for more than 1 year [20]. In addition, some alkaloid BSMs, such as berberine (BBR), were found to reduce PCSK9 mRNA and protein expression levels by inhibiting its transcription [21]. Several clinical studies also tested the efficacy of BBR in humans and suggested that administration of BBR may be a potential supplement to statin treatment for hypercholesterolemia [22]. Besides, compared with the currently monoclonal antibodies, small-molecule drugs have low hydrophilicity and are not easily degraded by gastrointestinal enzymes, which means a higher bioavailability.

\subsection{Diverse biological effects}

In fact, another feature of macromolecular drugs is that they usually target cell membrane receptors, while many BSMs can easily go through the cell membrane and target both intracellular and extracellular to regulate a variety of signaling pathways. This is what we call the diverse biological effects of BSMs.

\subsubsection{BSMs target the whole central law}

Different BSMs can regulate different steps of the central law. Some BSMs can go through the cell membrane and interact with DNA [23]. Others may bind RNA to regulate the downstream protein expression [24]. Still others can regulate different pathological progressions by targeting proteins such as some kinases in different signal pathway networks. Some BSMs can even bind both proteins and DNA to regulate human homeostasis. For example, puerarin, a natural small molecular product from herb Puerariae Radix (PR), is widely used in the treatment of cardiovascular diseases in China [25]. It was proved that to puerariae can down-regulate the expression of TLR4 protein and up-regulate he expression of C/EBP- $\beta$ protein to delay the occurrence of ventricular remodeling in in myocardial ischemia (MI) mice model [26]. And another research showed that it could also active the PPAR- $\gamma$ signaling and inhibit the NF- $\varkappa$ B signaling to reduce the inflammation in vivo and in vitro [27]. These were examples that puerariae involved in the regulation of signaling pathways by interacting with different proteins. While a new functional analysis showed that puerariae could also insert into the base of DNA and cause conformational changes in DNA to suppress cell proliferation [28], which might be a potential drug for cancer [29].

\subsubsection{Cross-talk between different BSMs in a pathological process}


In addition to the diversity of effective targets, participation in various disease pathological processes is also a manifestation of diverse biological effects of BSMs. In fact, there are two main forms that BSMs can exert in different cardiovascular disease.

First, different BSMs can constitute a cross-talk signaling network in a pathological process. Heart failure remains a considerable health burden as the common outcomes of various cardiovascular diseases. Restoring blood supply to the ischemic area and promoting angiogenesis were considered as novel therapies to improve cardiac function. As critical co-effectors of angiogenesis, gas cross-talk between nitric oxide (NO) and hydrogen sulfide $\left(\mathrm{H}_{2} \mathrm{~S}\right)$ was a good example. $\mathrm{NO}$ and $\mathrm{H}_{2} \mathrm{~S}$ not only had mutual interaction by changing the expression and activity of enzymes [30], but also mutually regulated the same downstream signaling pathways. Sirt1 was found to be a common downstream signal target of $\mathrm{NO}$ and $\mathrm{H}_{2} \mathrm{~S}$. After activated by NO and $\mathrm{H}_{2} \mathrm{~S}$, Sirt1 up-regulated p38 and ERK to participate in the angiogenesis by increasing the level of VEGF and accumulating cGMP [31]. Besides, they also exerted in a cross-talk in the cardiac remodeling procession of heart failure. After activated by the $\mathrm{K}^{+}$-ATP channels, $\mathrm{H}_{2} \mathrm{~S}$ was found to ameliorate L-NAME-induced cardiac remodeling and dysfunction by interacting with the NO-dependent pathway [32]. What's more, the cooperative action of these two gas molecules also impacted the angiogenesis and vasorelaxation. Inhibition of NO synthase (eNOS) can reduce the angiogenic response and vasodilation effect of $\mathrm{H} 2 \mathrm{~S}$, while inhibition of $\mathrm{H}_{2} \mathrm{~S}$ synthetase (CSE) can accelerate the degradation of cGMP, thereby reducing the vascular function of NO. Theses evidences means that the interaction between BSMs, such as $\mathrm{NO}$ and $\mathrm{H}_{2} \mathrm{~S}$, contributes as a cardioprotective factor.

\subsubsection{A BSM regulates multiple cardiovascular diseases}

Second, a small molecule may regulate multiple cardiovascular diseases. Adrenomedullin (ADM) was a small active peptide with only 52 amino acid residues derived from its precursor prepro-ADM. It was produced and released in vascular smooth muscle cells under the stimulation of many factors. Despite its small molecular weight, it could regulate various biological processes, such as vasodilation, myocardial hypertrophy, angiogenesis, fibrosis and inflammation, in different kinds of cardiovascular diseases. ADM can dilate the peripheral circulation and blood vessels in specific regions such as the brain, lung and kidney to increasing blood flow. Intravenous infusion of ADM was proved to be effective to reduce total peripheral resistance and blood pressure in humans and mice [33]. In cell culture experiment, ADM can promote NO synthesis to dilating endothelial cells and activate potassium channels by up-regulating cAMP to relax vascular smooth muscle cells [34]. One study also showed that chronic infusion of ADM had a protective effect in renal failure due to hypertension in a mice model [35]. All these evidences indicated that endogenous ADM may be involved in the pathological progress of hypertension and may have a protective effect on the target organs of hypertension. While, other studies suggested a vasculo-protective role of ADM against progression of atherosclerosis by inhibiting apoptosis and regulating proliferation. After the stimulation of platelet-derived growth factor (PDGF), ADM was shown to inhibit the proliferation of vascular smooth muscle cells [36]. Besides, ADM could promote the apoptosis of endothelial cells through cAMP-independent mechanism in both rat aortic endothelial cells and human umbilical vein endothelial cells [37]. And the aorta of apo-lipoprotein E (apoE)-knockout mice with ADM gene overexpression had significantly less fatty streak formation than those without ADM over-expression [38]. Moreover, some evidences even showed that plasma ADM levels can reflect the degree of endothelial injury in patients with atherosclerosis [39], which was likely to be a potential biomarker to detect atherosclerosis. What's more, ADM was also found to be associated with myocardial infarction and heart failure. Nakamura et al. showed that continuous infusion of ADM reduced the levels of mRNAs encoding ACE, p22-phox and urinary isoprostane to ameliorate left ventricular remodeling in an acute myocardial infarction rat model [40]. And chronic ADM administration was proved to attenuate transition of hypertrophy and improve survival in rats with heart failure [41].

\subsubsection{Other roles of BSMs in regulating the pathological process of CVD}

Besides, BSMs can play different roles at different stages of the same disease. Still take different pathological processes of heart failure as examples. Some BSMs derived from lipids such as Misoprostol were mainly involved in the inflammatory response and preservation of microcirculation after myocardial injury [42]. Other 
gas BSMs, such as $\mathrm{NO}$ and $\mathrm{H}_{2} \mathrm{~S}$, exerted in regulating cardiac remodeling after hypoxia [43]. After ventricular dilatation and cardiac hypertrophy by cardiac remodeling, uncoordinated beating of cardiomyocytes poses a high risk of fatal arrhythmias [44]. BSMs derived from metal ions, such as magnesium ions and potassium ions, regulated the cardiac ion channels and participated in myocardial electrophysiology $[45,46]$.

Also, the diversity biological effects of BSMs is also reflected in the fact that some BSMs previously treated for other diseases have also been proven to be useful for cardiovascular diseases. As a classic mitosis inhibitor, colchicine can bind to tubulin dimers and prevent them conversion to stop cells in the middle stage of mitosis. And it was found to exert an anti-inflammatory effect by interfering with lysosomal degranulation to reduce the activity, adhesion, and chemotaxis of neutrophils and to inhibit the migration of granulocytes to the inflammatory area. For this reason, colchicine was widely used in the treatment of gout as an inexpensive and oral administration [47]. However, researches on this alkaloid have been continuously expanded in recent years. It was indicated that colchicine may sustain the stability of plaque and inhibit various inflammatory factors to improve the clinical outcomes in patients with acute coronary syndromes. In the Low-Dose Colchicine (LoDoCo) trial, colchicine at a dose of $0.5 \mathrm{mg}$ once daily was shown to reduce the risk of longterm cardiovascular events in patients with clinically stable coronary [48]. To further evaluate the effects of colchicine among patients with a recent myocardial infarction the Colchicine Cardiovascular Outcomes Trial (COLCOT) was conducted and found that the low-dose colchicine significantly prevented patient from recurrent ischemic cardiovascular events than placebo did [49].

\subsection{Wide distribution of BSMs}

In addition, extensive tissue distribution is also an important feature of endogenous BSMs. Some molecules can be distributed in different tissues to regulate different effects. Many BSMs acting on the cardiovascular system were produced and released in myocardial cells and endothelial cells, while other BSMs were distributed in the nerve system. Neuropeptides, a class of BSMs derived from short peptides including neuropeptide Y (NPY), substance P (SP), calcitonin gene-related peptide (CGRP), vasoactive intestinal peptide (VIP) and others, were found to be distributed in sympathetic and parasympathetic nerves in the heart [50] to play an important role in regulating cardiovascular neuroendocrine. In addition to BSMs in the heart region, BSMs elsewhere have also been found to be involved in the development of cardiovascular disease.

Another example of small peptide was ghrelin, which was first found in the mice stomach and identified as a novel gastrointestinal hormone [51]. As an endogenous ligand for GH-secretagogue receptor, ghrelin activated the pathway of GH and its mediator, insulin-like growth factor-1 (IGF-1), both of which were anabolic hormones necessary for skeletal and myocardial growth and for metabolic homeostasis. Since GH/IGF-1 exerted effects on cardiac structure and function, ghrelin can affect the cardiovascular system through the elevation of plasma GH levels $[52,53]$. Although mainly distributed in stomach, ghrelin played a critical role in a variety of pathological processes of heart diseases. endogenous ghrelin and its receptor are associated with increased appetite. Cachexia, which was a catabolic state characterized by weight loss and muscle wasting, is associated with hormonal changed and cytokine activation in severely sick patients. Intravenous infusion of ghrelin was reported to increase food intake and body weight in healthy subjects [54] and to stimulate appetite and food intake in patients with congestive heart failure [55]. Therefore, it was conceivable that ghrelin administration could be a novel therapeutic approach for cachexia in humans [56]. Moreover, ghrelin exerted potent anti-inflammatory function to prevent atherosclerosis. It suppressed the production of proinflammatory cytokines, including IL- $1 \beta$, IL-6, and TNF- $\alpha$ and inhibits the activation of nuclear factor- $\chi \mathrm{B}(\mathrm{NF}-\varkappa \mathrm{B})$, a transcriptional factor regulating the gene expression of pro-inflammatory cytokines [57]. What's more, ghrelin potently inhibited sympathetic nerve which is often over-activated in cardiac diseases [58]. The effects of ghrelin on blood pressure, sympathetic nervous system activity, and mental stress responses were investigated in lean and overweight or obese individuals and it was found that stress-induced significant increase in these parameters were significantly reduced by $1 \mathrm{~h}$ intravenous infusion of ghrelin irrespective of obese phenotype [59]. In addition, administration of ghrelin significantly suppressed heart rate increase and ghrelin significantly suppressed plasma norepinephrine level in both humans and animals [58]. And it was demonstrated to dilate human artery through the endothelium- 
independent mechanism [60]. Ghrelin inhibited apoptosis of cultured cardiomyocytes and endothelial cells possibly through activation of extracellular signal-regulated kinase- $1 / 2$ and Akt serine kinases [61]. Moreover, ghrelin was proved to exert a direct positive inotropic effect on cardiomyocytes and increased myocardial contractility [62].

Besides small peptides, BSMs derived from small gas molecules were also distributed in many tissues and organs and plays an important regulatory role in the multi-organ cross-talk. Cystathionine gamma-lyase (CSE), a kind of $\mathrm{H}_{2} \mathrm{~S}$ synthetase, was found to be distributed in the heart, aorta, and other tissues [63] and helped $\mathrm{H}_{2} \mathrm{~S}$ impacted direct effects on the cardiovascular system [64]. However, $\mathrm{H}_{2} \mathrm{~S}$ was also widely distributed in the central nervous system. Its synthetase cystathionine- $\beta$-synthase (CBS) was widely expressed in glial Bergman and astrocytes [65], while its other synthetases CSE and 3-MST were also marked in neurons in the brain [66]. Compared with wild rats, endogenous $\mathrm{H}_{2} \mathrm{~S}$ levels in spontaneously hypertensive rats were significantly reduced, and the expression of CBS in the RVLM region of SHR rats was significantly down-regulated [67]. Chronic administration of NaHS $\left(\mathrm{H}_{2} \mathrm{~S}\right.$ donor) or SAM (CBS agonist) significantly reduced the average arterial pressure in spontaneously hypertensive rats [68], which suggested that $\mathrm{H}_{2} \mathrm{~S}$ in the brain might be involved in the development of spontaneous hypertension. At the same time, after injecting $\mathrm{NaHS}$ in the rostral ventrolateral medulla of rats, the $\mathrm{H}_{2} \mathrm{~S}$ can inhibit the excitability of sympathetic nerves by activating the ATP-sensitive $\mathrm{K}^{+}$channels [69]. All the evidences implicated that BSMs distributed in the nervous system may regulate the mechanism of blood pressure and exert in the pathogenesis of hypertension, which helped us build the molecular-cell-organ-integral strategies for modern cardiovascular disease research.

\subsection{Rapid synthesis and metabolism}

In fact, although BSMs played an important role in regulating various physiological and pathological progress of cardiovascular system, the concentrations of BSMs in the body were very low. There were many reasons for this problem. Some views suggested that indeed very small doses of BSMs could have immense biological effects in vitro tests. Urotensin II (UII) containing only 11 amino acids was identified as the most potent endogenous vasoconstrictor and its receptor UT was widely distributed in the heart, kidney and the central nervous system (CNS) [70, 71]. Only $10 \mathrm{nmol}$ dose of UII injected in intracerebroventricular (ICV) significantly improved the mean arterial pressure at 5 min than artificial cerebrospinal fluid injected in ICV in the conscious rat model [72]. And it was proved that the synthesis and metabolism of UII played an indispensable role in controlling the balance between vasoconstriction and blood pressure in human [73, 74]. Others believed that the low level of BSMs was due to insufficient detection methods since there were various kinds of BSMs and different BSMs needed different examination. But the rapid synthesis and metabolism of BSMs accounted most for this problem.

In the integrated regulating network, BSMs were detected to be produced instantly and exerted their function rapidly. And after the completed reaction, they were quickly metabolized to maintain the homeostasis. Therefore, it was really difficult to detect the true physiological concentration of BSMs in vivo. Sometimes we had to detect the modification of target proteins to re-evaluate the content of BSMs. It still remained unknown the exact concentration of $\mathrm{H}_{2} \mathrm{~S}$ since its sources and synthesis conditions were not be fully understood [75]. Some studies tried to detect sulfhydration, an oxidative posttranslational modification of target proteins caused by $\mathrm{H}_{2} \mathrm{~S}$, through different methods, such as the modified biotin switch assay [76], the maleimide assay [77], tag-switch technique [78], and mass spectroscopy. These methods provided an opportunity to capture the moment of synthesis and metabolism of $\mathrm{H}_{2} \mathrm{~S}$. However, sulfhydration was not the only reaction of $\mathrm{H}_{2} \mathrm{~S}$ and these methods were still time-lapse and had omissions.

This dilemma also occurred in the research of neuropeptides. Although some current antihypertensive drugs, such as ACEI and ART, targeted the renin-angiotensin system (RAS), this important blood pressure regulation network still had some unknown regulating mechanisms. In human brain, there were a series of neuropeptides derived from angiotensinogen. They widely involved in the physiological homeostasis of blood pressure and the pathological process of hypertension. However, an effective analytical method for detecting these small peptides of low concentrations in vivo was still lacking. To meet this challenge, a microanalytical capillary electrophoresis mass spectrometry assay was developed to depict a whole profile 
of different angiotensin in micro-sampled brain nuclei tissues [79]]. Despite the microanalytical assay, other methods were also developed to detect the neuropeptides. Somatostatin (SS), a well-known inhibitor of growth hormone, also involved in the regulation of cardiovascular system. Compared with intravenous administration of SS, SS given in certain brain nuclei increased the mean arterial blood pressure in rat models [80], which suggested that SS might exert its cardiovascular regulation through the central nervous system. But the discovery of this field didn't develop greatly due to the limited detecting methods. Recently, a biosensor based on Capacitive Micromachined Ultrasonic Transducer (CMUT) provided an opportunity to detecting low concentrations (pg ${ }^{n} \mathrm{ng} / \mathrm{ml}$ ) of SS in cerebrospinal fluid (CSF) [81], which could help us get novel insight into the regulation of SS. How to create visualizations for BSMs would be a tempting future direction and more high-sensitive probes would be developed to measure the real-time and dynamic changes of BSMs levels in cellular and tissues to reflect the real regulation network in cardiovascular homeostasis.

\section{New frontier of BSMs as new clinical application in CVD}

From the perspective of cardiovascular research, the current basic researches on BSMs mainly focus on the exact biological effects of different small molecules and how the BSMs regulate and modify the cell signal transduction and gene expression. From the above, we summarized five main characteristics of BSMs to depict a complex regulatory network of cardiovascular pathological processes formed by a variety of BSMs.

In recent years, new BSMs active in cardiovascular have been discovered continuously. Phoenixin (PNX), a novel predicted peptide first identified in 2013, was mainly expressed on the hypothalamus and it was regarded as a conservational regulating factor on reproductive homeostasis [82]. A recent study showed that PNX was also expressed on the mammalian heart and served as a cardioprotective factor. Although it consisted of 20 amino acids, PNX was shown to reduce the contractility and relaxation of heart in vitro test, and it could inhibit apoptosis to narrow the infarct size and accelerate the recovery of contractility compared with the ischemia/reperfusion (I/R) treat alone [83, 84], In addition, the experiments on the heart of obese rats showed the same result. All these evidences suggested that PNX was a novel regulating small molecule to exert in both the heart and metabolic disorders.

But merely depicting a static regulatory network of BSMs is not enough. How to make full use of the results of these basic studies of BSMs to transform into new therapies to improve the prognosis of patients with CVD occupies the main direction of future research. Next, we will discuss the progress and challenges of BSMs in drug development and other clinical applications in the era of big-data and bioinformation.

\subsection{Distance from BSMs to small-molecule drugs}

The most important reason to study BSMs is that it provides an opportunity to be a potential medicine and clinical intervention. Although the development of bio-macromolecule drugs represented by monoclonal antibodies, such as PD-1 [85], is quite predominant at present, small-molecular drug holds about an $80 \%$ share of the global drug market. And since the monoclonal antibodies are complicated to product and difficult to store, the heterogeneity among the effectiveness of the drugs obtained by different methods limits its wide use. Hence, the drugs derived from BSMs would a good alternative in cardiovascular fields.

\subsubsection{Two traditional processes for discovering small-molecule candidates}

The current research strategies for developing BSMs as drugs are quite limited. Figure 2 summarizes two traditional processes for developing small-molecule drugs in recent decades. One of them starts from the recognized BSMs, and then carries out experiments in different disease models and at different levels to explore the complex and variable regulatory networks of small molecules in order to find potential drug targets [86]. Usually, the BSMs in this research process are derived from natural products and have exact biological effects, which would reduce the research cycle and cost. However, it may also bring some negative effects. For example, the composition of natural products is complex, and it is difficult to directly determine which component plays a decisive role. At the same time, BSMs derived from natural products are often highly toxic and low bio-active with poor selectivity to targets. In addition, they might have poor stability and poor organic solubility during the preparation process. To solve this problem, various approaches combined 
with bioinformatics have been achieved with the development of computer-aided drug design (CADD) in recent years $[10,87,88]$. SwissTargetPrediction, a web server for target prediction of BSMs, was a good example [89]. It made full use of the natural concept that 'similar bioactive molecules are more likely to share similar targets' [90] to build a virtual platform, where the BSMs submitted would be compared with the BSMs existed in the sets to explore a potential target through molecular fingerprints and structural similarity measures with known ligands. And this method is widely used to choose the most sensitive BSMs among the homologous small molecules and it is more suitable for the optimization process in the later stages of drug development.

The other way to discover new drugs is based on the existing targets like kinases. With the development of high-throughput technology in recent years, this strategy has been combined with computers to virtually screen potential BSMs for the drug-target interactions (DTIs). And then the chosen BSMs will be subjected to subsequent pharmacological tests after being synthesized. This method is called structure-based drug design (SBDD). It demonstrates strong advantages on saving the time-consuming and cost of traditional biological experiments. However, the inevitable challenge it should confront is that the large amount of the high-dimensional data and background noise is always prepared to interfere the subsequent data analysis. In this field, different algorithms have been developed to solve this problem, such as molecular docking [91], structure-based virtual screening (SBVS) [92], and molecular dynamics (MD) [93] . AutoDock, a suite of integrated and interactive software was programmed to predict suitable small molecules binding with the known structure of the receptor based on the method of molecular docking, which saves both the timeconsuming and high cost for traditional chemical library screening [94].

\subsubsection{Next generation of how BSMs become drugs}

According to the previous, the two traditional research strategies have more or less been combined with computer methods. But their essence still starts from the known database, and mathematical models just play a supporting role in these strategies. How to make full use of the computer science to boost the small-molecule drug discovery is becoming a widespread concern. The establishment of artificial intelligence (AI) models and deep learning theories has gradually blurred the boundaries between data analysis and biological experiments [95], which will become the prototype of the next generation of small molecule research strategies. This kind of idea is not new, but most of the efforts made for it have not paid off. However, a new self-learning approach allows us to see the dawn of the next generation of drug design. Based on this strategy, an advanced AI-based sophisticated deep learning platform was developed to learn the structure and characteristics of BSMs by themselves, and then to predict the function of specific molecules [96]. A directed-message passing deep neural network model was adopted in this platform and it could accurately capture the bond messages about neighboring atoms and bonds accurately to determine the whole features of molecular structure. Based on this model, the platform was trained on a library of 2,335 molecules to get the capability to screen another 6,111 small molecules and rank these compounds according to the growth inhibition against E. coli. Then, halicin (SU3327), a c-Jun N-terminal kinase inhibitor potential for diabetes treatment [97], was selected to show a high antibacterial ability for A. baumannii and it was checked in the cellular and rat models. In view of the complex situation regarding widespread emergence of drug-resistant bacteria, this method to discovery new small molecule antibiotic was considered a subversion of traditional drug screening. What's more, this deep neural network model took only 3 days to screen 107 million BSMs entirely and 8 compounds were scored with antibacterial activity, two of which were shown highly reliable in biological test. This not only demonstrates the powerful screening capabilities of deep learning models, but also implies that this is a both efficient and economical novel drug development method. Of course, this model is not perfect, and it uses bacteriostatic ability as a scoring criterion in antibiotic research.

In fact, small molecule antibiotics design is just the beginning of the next era. Five grand challenges including appropriate datasets, new hypotheses, multi-objective optimization, cycle times reduction and research culture were still faced both the scientists and pharmaceutical companies [98]. Quantitative assessment of bacteriostatic capacity facilitates the scoring system for this platform. In the next step, it is necessary to further optimize the scoring mechanism to enhance the model's learning and screening capabilities in the 
application into screening the cardiovascular BSMs. How to learn from this deep learning models for the next drug development of cardiovascular BSMs is worthy of our concern.

\subsection{Other basic progress and clinical application of BSMs in CVD}

\subsubsection{Novel detecting technique boosts the understanding of BSMs in CVD}

Apart from the drug development in the study of BSMs, another issue that cannot be ignored is how to detect precisely the regulatory network of small molecules in the cardiovascular system. As described above, the nature of rapid synthesis and metabolism was regarded as a hinder for the BSMs research. Then appropriate detection methods can help us understand the regulatory network of small molecules and find the downstream targets of these BSMs. Many different methods are being developed to detect small molecules and their targets like proteins and RNAs [99-101]. Recently, a novel tag-free probe was designed to detect small molecules, target proteins, and their interactions simultaneously [102]. Quercetin, a BSMs derived from quercetin glycosides, was shown to prevent cardiac hypertrophy through the regulation network of proteasome inhibition and activation of proteasome-glycogen synthesis kinase $3 \alpha / \beta($ GSK-3 $\alpha / \beta)$ [103]. And its endogenous metabolites, 3,4-dihydroxyphenylacetic Acid (DOPAC), also played an anti-oxidation and anti-inflammation role in the amelioration of cardiovascular diseases and was found an increase of plasma in patients with congestive heart failure [104]. However, the specific mechanism of its interaction with downstream proteins is not completely understood. Now this situation will be reversed with the help of this new probe based on click chemistry [105]. After introduction of terminal alkyne through the Fischer esterification, DOPAC was changed into DPE with 2-propyn-1-ol and this change did not affect the conjugation of DOPAC or DPE with the target protein. While, DPE could be easily tagged by a click reaction called the copper(I)-catalyzed azide alkyne cycloaddition (CuAAC) [106] and this provided a fast detection method int subsequent pulldown experiments using biotin-tags. Under this method, Keap1 and aryl hydrocarbon receptor was identified as the target proteins of DOPAC. What's more, this tag-free probe could be combined with fluorescence tags to quantify the interaction between BSMs and their targets, and even be used to reveal the location where the BSMs exert their biological effects.

\subsubsection{Novel diagnostic strategies of BSMs-based biomarkers in CVD}

At the same time, with the development of detection technology, the clinical application of BSMs in cardiovascular diseases has gradually been widened. Many BSMs in human fluids can also be detected as biomarkers for various cardiovascular diseases.

Pathogenesis and diagnostic biomarkers for diseases can be discovered by metabolomic profiling of human fluids. If the various types of coronary artery disease (CAD) can be accurately characterized by metabolomics, effective treatment may be targeted without using unnecessary therapies and resources. Metabolic disorder is a characteristic of CAD, but there is lack of clinical detection of patient metabolism. A study was conducted to characterize metabolic imbalance by plasma BSMs in different types of CAD [107]. Differential metabolic small molecules including bile acids, amino acids, short chain acylcarnitines, tricarboxylic acid (TCA) cycle metabolites and phospholipids were shown to change in different CAD stages. By function enrichment and cluster analysis, these metabolic BSMs were indicated to alter different metabolic pathways. At the same time, 12 panels of specific metabolomics-based biomarkers were tested in the multicenter trial and highlighted a high sensitivity and specificity of diagnosing CAD at different stages. This showed the great potential clinical application of BSMs as biomarkers in cardiovascular disease.

Further, with the help of ultra-performance liquid chromatography and quadrupole time-of-flight mass spectrometry in the negative ion mode, a small molecule biomarker $\mathrm{N}$-acetylneuraminic acid (Neu5Ac) was identified to increase in patient plasma and shown a potential role in the pathological progression of CAD [108]. And the regulation network of this small molecule was associated with myocardial injury. By activating the Rho/Rho-associated coiled-coil containing protein kinase signaling pathway, Neu5Ac triggered RhoA and Cdc42-dependent myocardial injury. What's more, it was found that myocardial ischemia injury could be ameliorated after silencing neuraminidase-1, the regulatory enzyme of Neu5Ac, which served as a novel potential drug target for CAD treatment. 


\section{Conclusion}

In recent years, the understanding of BSMs in CVD has advanced significantly. Different types of BSMs including lipid, peptide, nucleic acid and others have been demonstrated to widely regulate all aspects of the development of cardiovascular disease. The five main features of BSMs summarized in this review depict a vivid and complex picture of integral interaction networks of BSMs in regulating the signal transduction pathways and gene expressions, which may have beneficial or harmful effects on the heart diseases. However, the researches of BSMs in cardiovascular physiology and pathology is still at a very early stage. The exact biological effects of many BSMs need to be expounded and many unknown small molecules remain to be discovered.

At the same time, the advancement of artificial intelligence and other information technology paves the way to discovering potential small-molecule drug candidates and expanding clinical applications of BSMs in cardiovascular disease. Although the prevalent bio-macromolecule drug development obscures the discovery of small-molecule drugs, novel paradigm of small-molecule drug design equipped with artificial intelligence and deep learning is gradually generating. Meanwhile, biomarkers of BSMs have been developed into the diagnosis of coronary heart disease, which provides new insights into the application of BSMs in cardiovascular diseases. What's more, many new approaches including the precise measurement of BSMs have been made to facilitate the further exploration of specific mechanism of BSMs regulation in the future.

\section{Reference}

[1] H. Thomas, J. Diamond, A. Vieco, S. Chaudhuri, E. Shinnar, S. Cromer, et al., Global Atlas of Cardiovascular Disease 2000-2016: The Path to Prevention and Control, Glob Heart 13(3) (2018) 143-163.

[2] G.B.D. Disease, I. Injury, C. Prevalence, Global, regional, and national incidence, prevalence, and years lived with disability for 328 diseases and injuries for 195 countries, 1990-2016: a systematic analysis for the Global Burden of Disease Study 2016, Lancet 390(10100) (2017) 1211-1259.

[3] M.R. Arkin, J.A. Wells, Small-molecule inhibitors of protein-protein interactions: progressing towards the dream, Nat Rev Drug Discov 3(4) (2004) 301-17.

[4] D.D. Baker, K.A. Alvi, Small-molecule natural products: new structures, new activities, Curr Opin Biotechnol 15(6) (2004) 576-83.

[5] T.F. Luscher, Heart failure: the cardiovascular epidemic of the 21st century, Eur Heart J 36(7) (2015) 395-7.

[6] G. Aubert, O.J. Martin, J.L. Horton, L. Lai, R.B. Vega, T.C. Leone, et al., The Failing Heart Relies on Ketone Bodies as a Fuel, Circulation 133(8) (2016) 698-705.

[7] J.G. Travers, F.A. Kamal, I. Valiente-Alandi, M.L. Nieman, M.A. Sargent, J.N. Lorenz, et al., Pharmacological and Activated Fibroblast Targeting of Gbetagamma-GRK2 After Myocardial Ischemia Attenuates Heart Failure Progression, J Am Coll Cardiol 70(8) (2017) 958-971.

[8] X.S. Zheng, T.F. Chan, H.H. Zhou, Genetic and genomic approaches to identify and study the targets of bioactive small molecules, Chem Biol 11(5) (2004) 609-18.

[9] Y.S. Ren, H.H. Li, J.C. Yao, Y.J. Tan, L.H. Pan, T. Peng, et al., Application quantitative proteomics approach to identify differentially expressed proteins associated with cardiac protection mediated by cycloastragenol in acute myocardial infarction rats, J Proteomics (2020) 103691.

[10] M.J. Keiser, B.L. Roth, B.N. Armbruster, P. Ernsberger, J.J. Irwin, B.K. Shoichet, Relating protein pharmacology by ligand chemistry, Nat Biotechnol 25(2) (2007) 197-206.

[11] G.D. Wright, Opportunities for natural products in 21(st) century antibiotic discovery, Nat Prod Rep 34(7) (2017) 694-701. 
[12] P.A. Lochhead, J.A. Tucker, N.J. Tatum, J. Wang, D. Oxley, A.M. Kidger, et al., Paradoxical activation of the protein kinase-transcription factor ERK5 by ERK5 kinase inhibitors, Nat Commun 11(1) (2020) 1383.

[13] C. Vitali, A.T. Remaley, M. Cuchel, Is Low-Density Lipoprotein Cholesterol the Key to Interpret the Role of Lecithin:Cholesterol Acyltransferase in Atherosclerosis?, Circulation 138(10) (2018) 1008-1011.

[14] E.A. Bohula, D.A. Morrow, R.P. Giugliano, M.A. Blazing, P. He, J.G. Park, et al., Atherothrombotic Risk Stratification and Ezetimibe for Secondary Prevention, J Am Coll Cardiol 69(8) (2017) 911-921.

[15] S.L. Pinkosky, S. Filippov, R.A. Srivastava, J.C. Hanselman, C.D. Bradshaw, T.R. Hurley, et al., AMPactivated protein kinase and ATP-citrate lyase are two distinct molecular targets for ETC-1002, a novel small molecule regulator of lipid and carbohydrate metabolism, J Lipid Res 54(1) (2013) 134-51.

[16] A.C. Goldberg, L.A. Leiter, E.S.G. Stroes, S.J. Baum, J.C. Hanselman, L.T. Bloedon, et al., Effect of Bempedoic Acid vs Placebo Added to Maximally Tolerated Statins on Low-Density Lipoprotein Cholesterol in Patients at High Risk for Cardiovascular Disease: The CLEAR Wisdom Randomized Clinical Trial, JAMA 322(18) (2019) 1780-1788.

[17] K.K. Ray, H.E. Bays, A.L. Catapano, N.D. Lalwani, L.T. Bloedon, L.R. Sterling, et al., Safety and Efficacy of Bempedoic Acid to Reduce LDL Cholesterol, N Engl J Med 380(11) (2019) 1022-1032.

[18] K.F. McClure, D.W. Piotrowski, D. Petersen, L. Wei, J. Xiao, A.T. Londregan, et al., Liver-Targeted Small-Molecule Inhibitors of Proprotein Convertase Subtilisin/Kexin Type 9 Synthesis, Angew Chem Int Ed Engl 56(51) (2017) 16218-16222.

[19] K. Guzik, K.M. Zak, P. Grudnik, K. Magiera, B. Musielak, R. Torner, et al., Small-Molecule Inhibitors of the Programmed Cell Death-1/Programmed Death-Ligand 1 (PD-1/PD-L1) Interaction via Transiently Induced Protein States and Dimerization of PD-L1, J Med Chem 60(13) (2017) 5857-5867.

[20] K.K. Ray, R.M. Stoekenbroek, D. Kallend, T. Nishikido, L.A. Leiter, U. Landmesser, et al., Effect of 1 or 2 Doses of Inclisiran on Low-Density Lipoprotein Cholesterol Levels: One-Year Follow-up of the ORION-1 Randomized Clinical Trial, JAMA Cardiol (2019).

[21] J. Cameron, T. Ranheim, M.A. Kulseth, T.P. Leren, K.E. Berge, Berberine decreases PCSK9 expression in HepG2 cells, Atherosclerosis 201(2) (2008) 266-73.

[22] J. Ju, J. Li, Q. Lin, H. Xu, Efficacy and safety of berberine for dyslipidaemias: A systematic review and meta-analysis of randomized clinical trials, Phytomedicine 50 (2018) 25-34.

[23] A.A. Almaqwashi, T. Paramanathan, I. Rouzina, M.C. Williams, Mechanisms of small molecule-DNA interactions probed by single-molecule force spectroscopy, Nucleic Acids Res 44(9) (2016) 3971-88.

[24] S.P. Velagapudi, M.G. Costales, B.R. Vummidi, Y. Nakai, A.J. Angelbello, T. Tran, et al., Approved Anti-cancer Drugs Target Oncogenic Non-coding RNAs, Cell Chem Biol 25(9) (2018) 1086-1094 e7.

[25] H. Xu, M. Zhao, S. Liang, Q. Huang, Y. Xiao, L. Ye, et al., The Effects of Puerarin on Rat Ventricular Myocytes and the Potential Mechanism, Sci Rep 6 (2016) 35475.

[26] S. Gao, L. Li, L. Li, J. Ni, R. Guo, J. Mao, et al., Effects of the combination of tanshinone IIA and puerarin on cardiac function and inflammatory response in myocardial ischemia mice, J Mol Cell Cardiol 137 (2019) 59-70.

[27] X. Li, T. Yuan, D. Chen, Y. Chen, S. Sun, D. Wang, et al., Cardioprotective Effects of Puerarin-V on Isoproterenol-Induced Myocardial Infarction Mice Is Associated with Regulation of PPAR-Upsilon/NFkappaB Pathway, Molecules 23(12) (2018).

[28] X. Chen, Z. He, X. Wu, D. Mao, C. Feng, J. Zhang, et al., Comprehensive study of the interaction between Puerariae Radix flavonoids and DNA: From theoretical simulation to structural analysis to functional analysis, Spectrochim Acta A Mol Biomol Spectrosc 231 (2020) 118109. 
[29] A. Tawani, S.K. Mishra, A. Kumar, Structural insight for the recognition of G-quadruplex structure at human c-myc promoter sequence by flavonoid Quercetin, Sci Rep 7(1) (2017) 3600.

[30] S. Kubo, I. Doe, Y. Kurokawa, H. Nishikawa, A. Kawabata, Direct inhibition of endothelial nitric oxide synthase by hydrogen sulfide: contribution to dual modulation of vascular tension, Toxicology 232(1-2) (2007) 138-46.

[31] Q. Hu, D. Wu, F. Ma, S. Yang, B. Tan, H. Xin, et al., Novel Angiogenic Activity and Molecular Mechanisms of ZYZ-803, a Slow-Releasing Hydrogen Sulfide-Nitric Oxide Hybrid Molecule, Antioxid Redox Signal 25(8) (2016) 498-514.

[32] S. Jin, X. Teng, L. Xiao, H. Xue, Q. Guo, X. Duan, et al., Hydrogen sulfide ameliorated L-NAME-induced hypertensive heart disease by the Akt/eNOS/NO pathway, Exp Biol Med (Maywood) 242(18) (2017) 18311841.

[33] N. Nagaya, T. Satoh, T. Nishikimi, M. Uematsu, S. Furuichi, F. Sakamaki, et al., Hemodynamic, renal, and hormonal effects of adrenomedullin infusion in patients with congestive heart failure, Circulation 101(5) (2000) 498-503.

[34] A.A. Voors, D. Kremer, C. Geven, J.M. Ter Maaten, J. Struck, A. Bergmann, et al., Adrenomedullin in heart failure: pathophysiology and therapeutic application, Eur J Heart Fail 21(2) (2019) 163-171.

[35] T. Nishikimi, Y. Mori, N. Kobayashi, K. Tadokoro, X. Wang, K. Akimoto, et al., Renoprotective effect of chronic adrenomedullin infusion in Dahl salt-sensitive rats, Hypertension 39(6) (2002) 1077-82.

[36] T. Horio, M. Kohno, H. Kano, M. Ikeda, K. Yasunari, K. Yokokawa, et al., Adrenomedullin as a novel antimigration factor of vascular smooth muscle cells, Circ Res 77(4) (1995) 660-4.

[37] M. Sata, M. Kakoki, D. Nagata, H. Nishimatsu, E. Suzuki, T. Aoyagi, et al., Adrenomedullin and nitric oxide inhibit human endothelial cell apoptosis via a cyclic GMP-independent mechanism, Hypertension 36(1) (2000) 83-8.

[38] Y. Imai, T. Shindo, K. Maemura, M. Sata, Y. Saito, Y. Kurihara, et al., Resistance to neointimal hyperplasia and fatty streak formation in mice with adrenomedullin overexpression, Arterioscler Thromb Vasc Biol 22(8) (2002) 1310-5.

[39] D.C. Bunton, M.C. Petrie, C. Hillier, F. Johnston, J.J. McMurray, The clinical relevance of adrenomedullin: a promising profile?, Pharmacol Ther 103(3) (2004) 179-201.

[40] R. Nakamura, J. Kato, K. Kitamura, H. Onitsuka, T. Imamura, K. Marutsuka, et al., Beneficial effects of adrenomedullin on left ventricular remodeling after myocardial infarction in rats, Cardiovasc Res 56(3) (2002) 373-80.

[41] T. Nishikimi, F. Yoshihara, S. Horinaka, N. Kobayashi, Y. Mori, K. Tadokoro, et al., Chronic administration of adrenomedullin attenuates transition from left ventricular hypertrophy to heart failure in rats, Hypertension 42(5) (2003) 1034-41.

[42] L. Zhu, C. Xu, X. Huo, H. Hao, Q. Wan, H. Chen, et al., The cyclooxygenase-1/mPGES-1/endothelial prostaglandin EP4 receptor pathway constrains myocardial ischemia-reperfusion injury, Nat Commun 10(1) (2019) 1888.

[43] P. Maneesai, S. Bunbupha, P. Potue, T. Berkban, U. Kukongviriyapan, V. Kukongviriyapan, et al., Hesperidin Prevents Nitric Oxide Deficiency-Induced Cardiovascular Remodeling in Rats via Suppressing TGF-beta1 and MMPs Protein Expression, Nutrients 10(10) (2018).

[44] J.H. Cho, R. Zhang, P.J. Kilfoil, R. Gallet, G. de Couto, C. Bresee, et al., Delayed Repolarization Underlies Ventricular Arrhythmias in Rats With Heart Failure and Preserved Ejection Fraction, Circulation 136(21) (2017) 2037-2050. 
[45] P. Zamani, V. Tan, H. Soto-Calderon, M. Beraun, J.A. Brandimarto, L. Trieu, et al., Pharmacokinetics and Pharmacodynamics of Inorganic Nitrate in Heart Failure With Preserved Ejection Fraction, Circ Res 120(7) (2017) 1151-1161.

[46] F. Romero, J. Palacios, I. Jofre, C. Paz, C.R. Nwokocha, A. Paredes, et al., Aristoteline, an IndoleAlkaloid, Induces Relaxation by Activating Potassium Channels and Blocking Calcium Channels in Isolated Rat Aorta, Molecules 24(15) (2019).

[47] B. Dasgeb, D. Kornreich, K. McGuinn, L. Okon, I. Brownell, D.L. Sackett, Colchicine: an ancient drug with novel applications, Br J Dermatol 178(2) (2018) 350-356.

[48] S.M. Nidorf, J.W. Eikelboom, C.A. Budgeon, P.L. Thompson, Low-dose colchicine for secondary prevention of cardiovascular disease, J Am Coll Cardiol 61(4) (2013) 404-410.

[49] J.C. Tardif, S. Kouz, D.D. Waters, O.F. Bertrand, R. Diaz, A.P. Maggioni, et al., Efficacy and Safety of Low-Dose Colchicine after Myocardial Infarction, N Engl J Med 381(26) (2019) 2497-2505.

[50] B.A. Habecker, M.E. Anderson, S.J. Birren, K. Fukuda, N. Herring, D.B. Hoover, et al., Molecular and cellular neurocardiology: development, and cellular and molecular adaptations to heart disease, J Physiol 594(14) (2016) 3853-75.

[51] D.E. Cummings, D.S. Weigle, R.S. Frayo, P.A. Breen, M.K. Ma, E.P. Dellinger, et al., Plasma ghrelin levels after diet-induced weight loss or gastric bypass surgery, N Engl J Med 346(21) (2002) 1623-30.

[52] N. Nagaya, M. Kojima, M. Uematsu, M. Yamagishi, H. Hosoda, H. Oya, et al., Hemodynamic and hormonal effects of human ghrelin in healthy volunteers, Am J Physiol Regul Integr Comp Physiol 280(5) (2001) R1483-7.

[53] Y. Date, N. Murakami, K. Toshinai, S. Matsukura, A. Niijima, H. Matsuo, et al., The role of the gastric afferent vagal nerve in ghrelin-induced feeding and growth hormone secretion in rats, Gastroenterology 123(4) (2002) 1120-8.

[54] A.M. Wren, L.J. Seal, M.A. Cohen, A.E. Brynes, G.S. Frost, K.G. Murphy, et al., Ghrelin enhances appetite and increases food intake in humans, J Clin Endocrinol Metab 86(12) (2001) 5992.

[55] N. Nagaya, J. Moriya, Y. Yasumura, M. Uematsu, F. Ono, W. Shimizu, et al., Effects of ghrelin administration on left ventricular function, exercise capacity, and muscle wasting in patients with chronic heart failure, Circulation 110(24) (2004) 3674-9.

[56] S. von Haehling, N. Ebner, M.R. Dos Santos, J. Springer, S.D. Anker, Muscle wasting and cachexia in heart failure: mechanisms and therapies, Nat Rev Cardiol 14(6) (2017) 323-341.

[57] W.G. Li, D. Gavrila, X. Liu, L. Wang, S. Gunnlaugsson, L.L. Stoll, et al., Ghrelin inhibits proinflammatory responses and nuclear factor-kappaB activation in human endothelial cells, Circulation 109(18) (2004) 2221-6.

[58] K. Matsumura, T. Tsuchihashi, K. Fujii, I. Abe, M. Iida, Central ghrelin modulates sympathetic activity in conscious rabbits, Hypertension 40(5) (2002) 694-9.

[59] E. Lambert, G. Lambert, C. Ika-Sari, T. Dawood, K. Lee, R. Chopra, et al., Ghrelin modulates sympathetic nervous system activity and stress response in lean and overweight men, Hypertension 58(1) (2011) 43-50.

[60] H. Okumura, N. Nagaya, M. Enomoto, E. Nakagawa, H. Oya, K. Kangawa, Vasodilatory effect of ghrelin, an endogenous peptide from the stomach, J Cardiovasc Pharmacol 39(6) (2002) 779-83.

[61] G. Baldanzi, N. Filigheddu, S. Cutrupi, F. Catapano, S. Bonissoni, A. Fubini, et al., Ghrelin and des-acyl ghrelin inhibit cell death in cardiomyocytes and endothelial cells through ERK1/2 and PI 3-kinase/AKT, J Cell Biol 159(6) (2002) 1029-37. 
[62] Q. Sun, Y. Ma, L. Zhang, Y.F. Zhao, W.J. Zang, C. Chen, Effects of GH secretagogues on contractility and $\mathrm{Ca} 2+$ homeostasis of isolated adult rat ventricular myocytes, Endocrinology 151(9) (2010) 4446-54.

[63] G. Yang, L. Wu, B. Jiang, W. Yang, J. Qi, K. Cao, et al., H2S as a physiologic vasorelaxant: hypertension in mice with deletion of cystathionine gamma-lyase, Science 322(5901) (2008) 587-90.

[64] S. Mani, H. Li, A. Untereiner, L. Wu, G. Yang, R.C. Austin, et al., Decreased endogenous production of hydrogen sulfide accelerates atherosclerosis, Circulation 127(25) (2013) 2523-34.

[65] T. Morikawa, M. Kajimura, T. Nakamura, T. Hishiki, T. Nakanishi, Y. Yukutake, et al., Hypoxic regulation of the cerebral microcirculation is mediated by a carbon monoxide-sensitive hydrogen sulfide pathway, Proc Natl Acad Sci U S A 109(4) (2012) 1293-8.

[66] N. Shibuya, M. Tanaka, M. Yoshida, Y. Ogasawara, T. Togawa, K. Ishii, et al., 3-Mercaptopyruvate sulfurtransferase produces hydrogen sulfide and bound sulfane sulfur in the brain, Antioxid Redox Signal 11(4) (2009) 703-14.

[67] H. Yu, H. Xu, X. Liu, N. Zhang, A. He, J. Yu, et al., Superoxide Mediates Depressive Effects Induced by Hydrogen Sulfide in Rostral Ventrolateral Medulla of Spontaneously Hypertensive Rats, Oxid Med Cell Longev 2015 (2015) 927686.

[68] J. Li, X. Teng, S. Jin, J. Dong, Q. Guo, D. Tian, et al., Hydrogen sulfide improves endothelial dysfunction by inhibiting the vicious cycle of NLRP3 inflammasome and oxidative stress in spontaneously hypertensive rats, J Hypertens 37(8) (2019) 1633-1643.

[69] Q. Guo, S. Jin, X.L. Wang, R. Wang, L. Xiao, R.R. He, et al., Hydrogen sulfide in the rostral ventrolateral medulla inhibits sympathetic vasomotor tone through ATP-sensitive K+ channels, J Pharmacol Exp Ther 338(2) (2011) 458-65.

[70] H. Vaudry, J. Leprince, D. Chatenet, A. Fournier, D.G. Lambert, J.C. Le Mevel, et al., International Union of Basic and Clinical Pharmacology. XCII. Urotensin II, urotensin II-related peptide, and their receptor: from structure to function, Pharmacol Rev 67(1) (2015) 214-58.

[71] S.A. Douglas, E.H. Ohlstein, Human urotensin-II, the most potent mammalian vasoconstrictor identified to date, as a therapeutic target for the management of cardiovascular disease, Trends Cardiovasc Med 10(6) (2000) 229-37.

[72] Y. Lin, T. Tsuchihashi, K. Matsumura, I. Abe, M. Iida, Central cardiovascular action of urotensin II in conscious rats, J Hypertens 21(1) (2003) 159-65.

[73] S.A. Douglas, A.C. Sulpizio, V. Piercy, H.M. Sarau, R.S. Ames, N.V. Aiyar, et al., Differential vasoconstrictor activity of human urotensin-II in vascular tissue isolated from the rat, mouse, dog, pig, marmoset and cynomolgus monkey, Br J Pharmacol 131(7) (2000) 1262-74.

[74] B. Ross, K. McKendy, A. Giaid, Role of urotensin II in health and disease, Am J Physiol Regul Integr Comp Physiol 298(5) (2010) R1156-72.

[75] B.D. Paul, S.H. Snyder, Modes of physiologic H2S signaling in the brain and peripheral tissues, Antioxid Redox Signal 22(5) (2015) 411-23.

[76] A.K. Mustafa, M.M. Gadalla, N. Sen, S. Kim, W. Mu, S.K. Gazi, et al., H2S signals through protein S-sulfhydration, Sci Signal 2(96) (2009) ra72.

[77] N. Sen, B.D. Paul, M.M. Gadalla, A.K. Mustafa, T. Sen, R. Xu, et al., Hydrogen sulfide-linked sulfhydration of NF-kappaB mediates its antiapoptotic actions, Mol Cell 45(1) (2012) 13-24.

[78] D. Zhang, I. Macinkovic, N.O. Devarie-Baez, J. Pan, C.M. Park, K.S. Carroll, et al., Detection of protein S-sulfhydration by a tag-switch technique, Angew Chem Int Ed Engl 53(2) (2014) 575-81. 
[79] C. Lombard-Banek, Z. Yu, A.P. Swiercz, P.J. Marvar, P. Nemes, A microanalytical capillary electrophoresis mass spectrometry assay for quantifying angiotensin peptides in the brain, Anal Bioanal Chem 411(19) (2019) 4661-4671.

[80] M.R. Brown, Somatostatin-28 effects on central nervous system regulation of vasopressin secretion and blood pressure, Neuroendocrinology 47(6) (1988) 556-62.

[81] S. Lee, G. Eom, I. Yoon, S. Park, G. Kook, M.K. Kim, et al., Capacitive Micromachined Ultrasonic Transducer (CMUT)-based Biosensor for Detection of Low Concentration Neuropeptide, Conf Proc IEEE Eng Med Biol Soc 2018 (2018) 2897-2900.

[82] G.L. Yosten, R.M. Lyu, A.J. Hsueh, O. Avsian-Kretchmer, J.K. Chang, C.W. Tullock, et al., A novel reproductive peptide, phoenixin, J Neuroendocrinol 25(2) (2013) 206-15.

[83] C. Rocca, F. Scavello, M.C. Granieri, T. Pasqua, N. Amodio, S. Imbrogno, et al., Phoenixin-14: detection and novel physiological implications in cardiac modulation and cardioprotection, Cell Mol Life Sci 75(4) (2018) 743-756.

[84] R.M. Lyu, A. Cowan, Y. Zhang, Y.H. Chen, S.L. Dun, J.K. Chang, et al., Phoenixin: a novel brain-gutskin peptide with multiple bioactivity, Acta Pharmacol Sin 39(5) (2018) 770-773.

[85] L. Ratner, T.A. Waldmann, M. Janakiram, J.E. Brammer, Rapid Progression of Adult T-Cell LeukemiaLymphoma after PD-1 Inhibitor Therapy, N Engl J Med 378(20) (2018) 1947-1948.

[86] W.L. Jorgensen, Efficient drug lead discovery and optimization, Acc Chem Res 42(6) (2009) 724-33.

[87] S. Ziegler, V. Pries, C. Hedberg, H. Waldmann, Target identification for small bioactive molecules: finding the needle in the haystack, Angew Chem Int Ed Engl 52(10) (2013) 2744-92.

[88] Y. Luo, X. Zhao, J. Zhou, J. Yang, Y. Zhang, W. Kuang, et al., A network integration approach for drug-target interaction prediction and computational drug repositioning from heterogeneous information, Nat Commun 8(1) (2017) 573.

[89] D. Gfeller, A. Grosdidier, M. Wirth, A. Daina, O. Michielin, V. Zoete, SwissTargetPrediction: a web server for target prediction of bioactive small molecules, Nucleic Acids Res 42(Web Server issue) (2014) W32-8.

[90] M. Campillos, M. Kuhn, A.C. Gavin, L.J. Jensen, P. Bork, Drug target identification using side-effect similarity, Science 321(5886) (2008) 263-6.

[91] X. Zhang, D. Wang, X. Ren, A.G. Atanasov, R. Zeng, L. Huang, System Bioinformatic Approach Through Molecular Docking, Network Pharmacology and Microarray Data Analysis to Determine the Molecular Mechanism Underlying the Effects of Rehmanniae Radix Praeparata on Cardiovascular Diseases, Curr Protein Pept Sci 20(10) (2019) 964-975.

[92] T. Ran, W. Li, B. Peng, B. Xie, T. Lu, S. Lu, et al., Virtual Screening with a Structure-Based Pharmacophore Model to Identify Small-Molecule Inhibitors of CARM1, J Chem Inf Model 59(1) (2019) 522-534.

[93] S. Decherchi, G. Bottegoni, A. Spitaleri, W. Rocchia, A. Cavalli, BiKi Life Sciences: A New Suite for Molecular Dynamics and Related Methods in Drug Discovery, J Chem Inf Model 58(2) (2018) 219-224.

[94] S. Forli, R. Huey, M.E. Pique, M.F. Sanner, D.S. Goodsell, A.J. Olson, Computational protein-ligand docking and virtual drug screening with the AutoDock suite, Nat Protoc 11(5) (2016) 905-19.

[95] D.M. Camacho, K.M. Collins, R.K. Powers, J.C. Costello, J.J. Collins, Next-Generation Machine Learning for Biological Networks, Cell 173(7) (2018) 1581-1592.

[96] J.M. Stokes, K. Yang, K. Swanson, W. Jin, A. Cubillos-Ruiz, N.M. Donghia, et al., A Deep Learning Approach to Antibiotic Discovery, Cell 180(4) (2020) 688-702 e13. 
[97] S. Jang, L.R. Yu, M.A. Abdelmegeed, Y. Gao, A. Banerjee, B.J. Song, Critical role of c-jun N-terminal protein kinase in promoting mitochondrial dysfunction and acute liver injury, Redox Biol 6 (2015) 552-564.

[98] P. Schneider, W.P. Walters, A.T. Plowright, N. Sieroka, J. Listgarten, R.A. Goodnow, Jr., et al., Rethinking drug design in the artificial intelligence era, Nat Rev Drug Discov (2019).

[99] A. McFedries, A. Schwaid, A. Saghatelian, Methods for the elucidation of protein-small molecule interactions, Chem Biol 20(5) (2013) 667-73.

[100] X. Chen, N.N. Guan, Y.Z. Sun, J.Q. Li, J. Qu, MicroRNA-small molecule association identification: from experimental results to computational models, Brief Bioinform (2018).

[101] H.Y. Hwang, T.Y. Kim, M.A. Szasz, B. Dome, J. Malm, G. Marko-Varga, et al., Profiling the Protein Targets of Unmodified Bio-Active Molecules with Drug Affinity Responsive Target Stability and Liquid Chromatography/Tandem Mass Spectrometry, Proteomics (2020) e1900325.

[102] S. Nakashima, Z. Liu, Y. Yamaguchi, S. Saiki, S. Munemasa, T. Nakamura, et al., A novel tag-free probe for targeting molecules interacting with a flavonoid catabolite, Biochem Biophys Rep 7 (2016) 240-245.

[103] K. Chen, M. Rekep, W. Wei, Q. Wu, Q. Xue, S. Li, et al., Quercetin Prevents In Vivo and In Vitro Myocardial Hypertrophy Through the Proteasome-GSK-3 Pathway, Cardiovasc Drugs Ther 32(1) (2018) $5-21$.

[104] G. Mercuro, Z.L. Rossetti, C.A. Rivano, A. Cherchi, G.L. Gessa, Marked increase of plasma 3,4dihydroxyphenylacetic acid in congestive heart failure, Am Heart J 108(6) (1984) 1588-9.

[105] C. Deane, Bioorthogonal chemistry: Click on, click off, Nat Chem Biol 13(10) (2017) 1057.

[106] G.R. Abel, Jr., Z.A. Calabrese, J. Ayco, J.E. Hein, T. Ye, Measuring and Suppressing the Oxidative Damage to DNA During Cu(I)-Catalyzed Azide-Alkyne Cycloaddition, Bioconjug Chem 27(3) (2016) 698704 .

[107] Y. Fan, Y. Li, Y. Chen, Y.J. Zhao, L.W. Liu, J. Li, et al., Comprehensive Metabolomic Characterization of Coronary Artery Diseases, J Am Coll Cardiol 68(12) (2016) 1281-93.

[108] L. Zhang, T.T. Wei, Y. Li, J. Li, Y. Fan, F.Q. Huang, et al., Functional Metabolomics Characterizes a Key Role for N-Acetylneuraminic Acid in Coronary Artery Diseases, Circulation 137(13) (2018) 1374-1390.

\section{Tables}

Table 1. Chemical classification of bioactive small molecules (BSMs).

\begin{tabular}{ll}
\hline Chemical classification & Examples associated with cardiovascular diseases \\
\hline Gas molecules & Nitric oxide (NO), Carbon monoxide (CO), Hydrogen sulfide \\
Lipid molecules & Lysophosphatidic acid (LPA), Epoxyeicosatrienoic acids (EE \\
Small molecular proteins and peptides & Endothelin-1 (ET-1), Adrenomedullin (AM), Ghrelin, Adipor \\
Active amino acids and its derivatives and amine substance & L-Arginine, Homocysteine (Hcy), Taurine \\
Metal ion & Calcium ions, Magnesium ions, Iron ions, Copper ions \\
Alkaloids & Colchicine - Berberine(BBR), Rescinnamine \\
Vitamin & Vitamin A, Vitamin D, Vitamin C \\
Nucleotide & Oligonucleotides, sequence-based design of bioactive small mo \\
\hline
\end{tabular}

Table 2. Comparison of main characteristics of small molecule drugs and biological macromolecule drugs.

\begin{tabular}{lll}
\hline Property & Small molecule drugs & Biological macromolecule drugs \\
\hline Relative molecular weight & Most are $<1000 \mathrm{Da}$ & Most are $>1000 \mathrm{Da}$
\end{tabular}




\begin{tabular}{lll}
\hline Property & Small molecule drugs & Biological macromolecule drugs \\
\hline Composition & Single component & Multicomponent \\
Physical and chemical properties & Distinct & Complex \\
Synthetic method & Chemical synthesis & Cell generation and isolation \\
Stability and sensitivity & Stable and Insensitive to heat & Sensitive to heat and shear \\
Administration routes & Usually oral & Parenteral \\
Absorption routes & By capillaries & By lymphatic vessels \\
Half-life & Variable from hours to 1 day & Variable from hours to weeks \\
Distribution & Organs and tissues & Plasma and extracellular fluid \\
Immunogenicity & Non-antigenic & Antigenic \\
\hline
\end{tabular}

\section{Figure Legends}

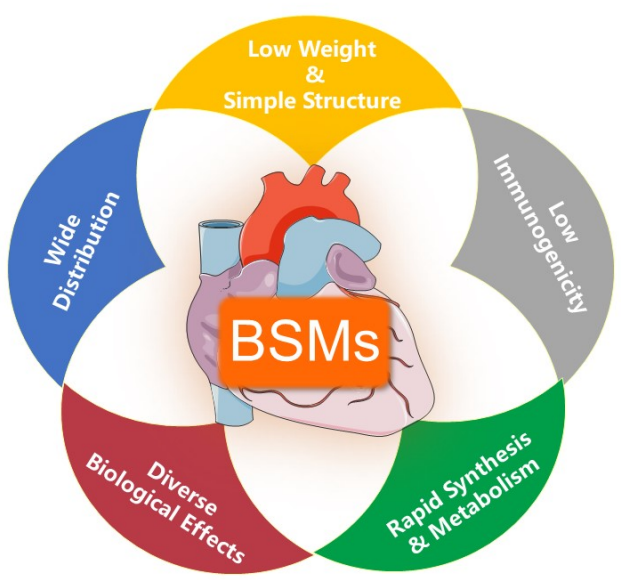

Figure 1. Five main characteristics of bioactive small molecules (BSMs) in cardiovascular diseases.

Parts of the single elements used to construct the figure were taken from https://smart.servier.com/ and minor modifications (e.g., color) were applied.

A)

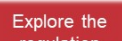

regulation
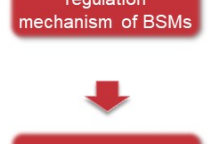

Confirm the direct targets of BSMs

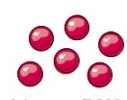

A known BSMs

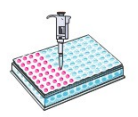

Molecularlevel

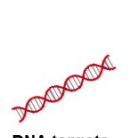

DNA targets
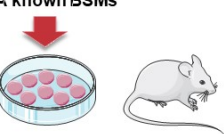

Cellular level Animal model 5

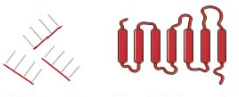

RNA targets Proteintargets

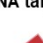

B)

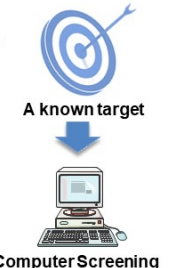

ComputerScreening

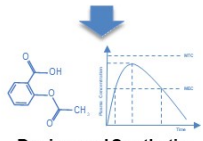

Design and Synthetic

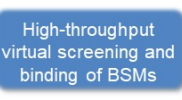

binding of BSMs

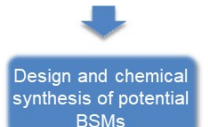


Figure 2. Two traditional processes of small-molecule drugs development.

A. BSMs-based strategy for drug development, B. Target-based strategy for drug development

Parts of the single elements used to construct the figure were taken from https://smart.servier.com/ and minor modifications (e.g., color) were applied.

\section{Hosted file}

Table 1.docx available at https://authorea.com/users/317426/articles/447525-an-integral-approachfor-understanding-the-bioactive-small-molecules-in-cardiovascular-diseases

\section{Hosted file}

Table 2.docx available at https://authorea.com/users/317426/articles/447525-an-integral-approachfor-understanding-the-bioactive-small-molecules-in-cardiovascular-diseases

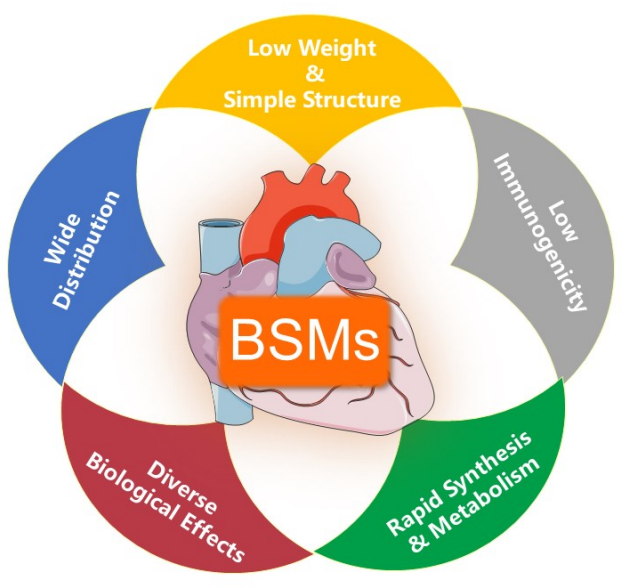

A)

\section{Explore the}

chanism of BSMs

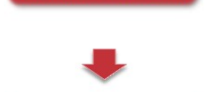

Confirm the direct targets of BSMs
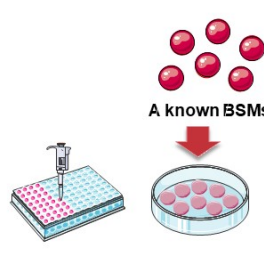

A known BSMs

Molecular level

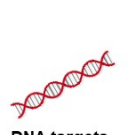

DNA targets

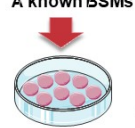

Cellular level Animal model $\checkmark$

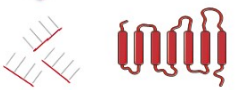

RNA targets Proteintargets

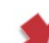

B)

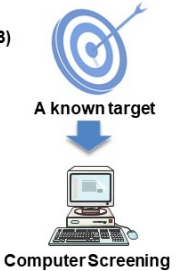

ComputerScreening

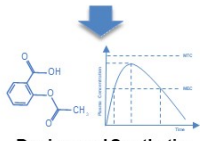

Design and Synthetic

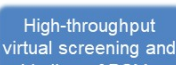

binding

binding of BSMs

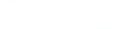

Design and chemical

synthesis of potential

BSMs
BSo 